\title{
Desafíos de la Implementación de la Estrategia de Negocio
}

doi: 10.33264/rpa.202101-04

Ulises Bacho Gahona

Facultad de Administración UNIACC

\section{Resumen}

Obstáculos y factores que inciden en la implementación de una estrategia de negocios son analizados en el presente artículo. Mediante una revisión de la literatura pertinente, se describe un breve marco conceptual que identifica los principales obstáculos a la implementación estratégica exitosa. En seguida, se analizan factores que posibilitan implementar correctamente la estrategia de negocio. Se concluye, con el desafío de gestionar de manera interdependiente los factores analizados.

Palabras claves: Implementación de la estrategia, ejecución de la estrategia, estrategia de negocio.

\section{Abstract}

Obstacles and factors that affect the implementation of a business strategy are analyzed in this article. Through a review of the relevant literature, a brief conceptual framework is described that identifies the main obstacles to successful strategic implementation. Next, factors that make it possible to correctly implement the business strategy are analyzed. It concludes, with the challenge of managing the factors analyzed interdependently.

Keywords: Strategy implementation, strategy execution, business strategy 


\section{Introducción}

La empresa en su aspiración de obtener y mantener una ventaja competitiva realiza un esfuerzo importante por comprender las tendencias emergentes del mercado, identificar las oportunidades y amenazas del macroentorno y reconocer la dinámica de las variables que modelan la industria en la que compite. Este análisis estratégico, permite, entre otros, formular una estrategia de negocio. Sin embargo, la etapa que sigue es la implementación de la estrategia, y es aquí donde aparece un desafío relevante.

La distinción entre la formulación de la estrategia y su implementación ofrece una oportunidad para ir conociendo el tipo de decisiones, ámbito y alcance de cada uno de los conceptos (Favaro, 2015, p. 25). El tema de la implementación de la estrategia ha sido revisado desde una perspectiva normativa (David et al., 2020; Dess et al., 2019; Gamble et al., 2020; Hill, 2019; Hitt et al., 2020). Así, se establecen pasos y etapas que se deberían seguir a fin de lograr una implementación exitosa de la estrategia.

No obstante, el hecho de que la estrategia "supuestamente" este bien formulada no es garantía de que ella se pueda implementar exitosamente (Barth \& De Beer, 2018; Kolbusa, 2013; Kotler et al., 2008).La implementación de una nueva estrategia de negocios, sin duda, trae cambios a la organización. En ese contexto, los autores Vyas y Westervel (2015, p. 13) plantean que las razones más esgrimidas para el fracaso de una iniciativa de transformación empresarial es la ejecución ineficiente con un 40\%, seguida de las limitaciones y presupuesto con un $35 \%$.

En general, toda iniciativa de transformación empresarial supone depender de las innumerables conexiones e interdependencias de los colaboradores de la organización. Así, una de las causas de la mala implementación de la estrategia es asumir que la alta dirección es el ente pensante y el resto sólo sigue órdenes del cerebro sin ninguna posibilidad ni opción, esta temática está muy bien tratada en Martin (2010).

La idea de que la implementación es el lado táctico de la estrategia, y por tanto sea un asunto que se puede delegar es errada. En efecto, tal como lo señalan los autores Bossidy y Charan (2002) la implementación no es sólo táctica, sino que también es una disciplina y un sistema en el cual el rol de líder es primordial. Tal como señalan Porter et al. (2007), un posible obstáculo a la buena implementación de la estrategia de negocios es que no todos los líderes tienen las capacidades y habilidades para lograr semejante tarea. Por tanto, seleccionar a las personas adecuadas para llevar a cabo la implementación es una dimensión del éxito de la implementación. 
En consecuencia, tener en consideración las potenciales barreras de la ejecución de la estrategia posiblemente contribuya a lograr la misión y objetivos estratégicos de la organización. Sin embargo, reconocer el cómo diversas fuerzas se combinan y relacionan, en el día a día organizacional, es un primer paso para una buena implementación de la estrategia de negocios. En este artículo se argumenta que, el desafío de la implementación de la estrategia implica considerar diversos factores que interactúan constantemente en la implementación exitosa de una estrategia de negocios. El artículo se inicia con una breve descripción del concepto de implementación de la estrategia de negocio. En seguida, se describen algunos obstáculos a la implementación. A continuación, se identifican factores que intervienen en la implementación de la estrategia. Finalmente, se establecen los principales desafíos que actualmente presenta la implementación de la estrategia de negocios.

\section{Implementación de la estrategia de negocios}

En el ámbito de la estrategia de negocio, la formulación y la implementación de la estrategia forma una pareja de conceptos esenciales para lograr un desempeño organizacional superior. En cuanto a la formulación de la estrategia, ella es el resultado del pensamiento estratégico organizacional, usualmente liderado por la alta dirección. Se fundamenta en un análisis o escaneo de los factores del macroentorno, a fin de aprovechar las oportunidades existentes y mitigar las posibles amenazas encontradas (David et al., 2020; Hill, 2019).Adicionalmente, este escaneo se acompaña por un análisis del microentorno el cual permite evidenciar las fuerzas que interactúan en la industria y que permiten determinar la atractividad de la industria en la cual la empresa participa y reconocer a los competidores cercanos (Porter, 1980).

No obstante, en la formulación también se contempla la posibilidad de dar cuenta de las oportunidades emergentes del mercado, las cuales muchas veces no están en el horizonte de planeación de la estrategia (Mintzberg, 2013). El marco conceptual planteado en párrafos previos corresponde a la visión de las escuelas de pensamiento de tipo prescriptivo en torno al tema. En efecto, de acuerdo con Mintzberg (2009) existirían a lo menos diez escuelas de pensamiento que investigan el fenómeno de la estrategia en acción.

La implementación de la estrategia es el siguiente paso en la búsqueda de una ventaja competitiva sostenible. Esta etapa es más complicada y altamente demandante de tiempo, un recurso cada vez más escaso, habida cuenta de la alta competitividad y dinámica de cambios de los mercados actuales. La implementación de la estrategia da cuenta de todas las decisiones y actividades necesarias para traducir la estrategia al plano de la realidad contingente. 
Si la ejecución de la estrategia es un proceso sistemático de exponer la realidad y actuar sobre ella (Bossidy \& Charan, 2002, p. 22), entonces el rol del liderazgo estratégico es esencial. En efecto, en él recae la responsabilidad de hacer las preguntas incomodas que los demás deberán responder, administrar el proceso de compartir, analizar y debatir toda la información disponible y hacer las correcciones pertinentes para que la implementación de la estrategia de negocio sea exitosa (Bossidy \& Charan, 2002, p. 25).

A pesar del rol del liderazgo estratégico, en la implementación de la estrategia está siempre presente la cultura organizacional y los colaboradores que la componen. Así, podremos encontrar empresas tales como 3M, Intel, Pfizer y Procter and Gamble entre otras que, reconocidamente han logrado construir y gestionar una fuerte cultura organizacional orientada a la innovación y altos niveles de desempeño.

\section{Algunos obstáculos a la implementación de la estrategia}

Desde la perspectiva de la ejecución de proyectos, usualmente se establecen tres aspectos que posiblemente impidan su consecución exitosa: tiempo y recursos, actividades en vez de resultado e intereses personales versus los objetivos organizacionales (Kolbusa, 2013, p. 25). En consecuencia, es muy difícil tratar de implementar una estrategia si no se cuentan con los recursos necesarios para llevarla cabo. Así, los recursos tangibles físicos como las maquinarias, edificios y materias primas, en conjunto con los recursos financieros de capital de trabajo, disponible para inversión entre otros deben estar disponibles para la implementación de la estrategia.

En relación con los recursos intangibles humanos, esto es, colaboradores con las capacidades y habilidades requeridas para llevar a cabo las acciones necesarias de ejecución estratégica en conjunto con lo intangible no humano tales como la imagen de marca, reputación y posicionamiento de la empresa forman parte de una mezcla general de recursos que se deben disponer al momento de implementar una estrategia de negocios.

Un horizonte de tiempo fuera de las posibilidades reales de cumplir por parte de la empresa no solo estresa en demasía a la organización, sino que, también afecta la calidad y efectividad de la implementación de la estrategia. La concentración excesiva en desmedro de los resultados es también un factor para considerar en la implementación de la estrategia. Aquí, el aporte inicial de medio siglo de Peter F. Drucker (2017) sigue vigente, lo que no se puede medir no se puede gestionar. Así, los autores Norton y Kaplan (2001, 2004, 2006, 2008; 1998) han puesto a disposición su famoso cuadro de mando integral como una manera de medir y controlar los resultados de la implementación de la estrategia. 
El desalineamiento entre los objetivos personales y los objetivos organizacionales es un tema que claramente afecta la implementación eficaz. Este conflicto de interés es dañino y muchas veces no es tan fácil de detectar (Whetten \& Cameron, 2020).

Toda nueva estrategia implica cambio y con ello aparece fenómenos como el miedo a lo desconocido y la incertidumbre que viene aparejada con lo nuevo. Kotter (2012) advierte de este fenómeno en las organizaciones y provee una camino a seguir para superar este obstáculo.

En el mundo competitivo actual, la aparición de tecnologías disruptivas puede borrar de una plumada todos los éxitos pasados de la organización, tal como sucedió en su oportunidad con la famosa y exitosas empresas Kodak y Blockbuster. En consecuencia, la parálisis paradigmática es un gran obstáculo a la implementación de la estrategia (Barker, 1993).

\section{Factores que intervienen en la implementación de la estrategia}

Los factores que intervienen en la implementación de la estrategia son diversos e interdependientes. Sin embargo, en la Figura 1 se identifican los principales de ellos, los cuales se explicarán a continuación.

Figura 1: Factores Principales que intervienen en la Implementación de la Estrategia.

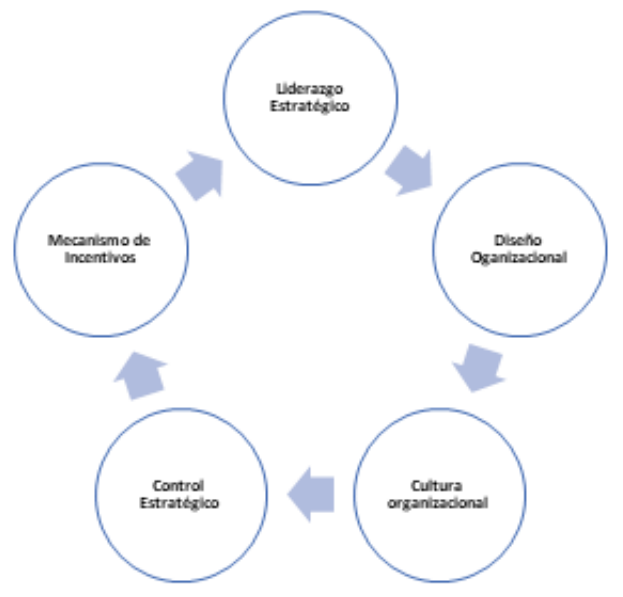

Fuente: En base a Radomska, J. (2014). The role of managers in effective strategy implementation. International Journal of Contemporary Management, 13(3), 77-85 y Barth, A. L., \& De Beer, W. (2018).

Performance management success: a best practices and implementation guide for leaders and managers of all organizations. Springer. 
El liderazgo estratégico es usualmente señalado como esencial al momento de intentar llevar el plan estratégico de la empresa a la realidad (Bennis \& Nanus, 1997; Bennis \& Townsend, 2005; Radomska, 2014). Si la intención del plan estratégico es cambiar lo hasta aquí realizado, entonces de acuerdo a Bennis (1997) el liderazgo es la capacidad para traducir la intención en realidad y sostenerla. En ello el uso sabio del poder es fundamental.

La empresa Space $X$, hasta la fecha ha logrado impresionantes objetivos al enviar exitosamente una serie de cohetes al espacio exterior. Adicionalmente, la empresa Tesla se ha trasformado en una empresa exitosa en la fabricación de automóviles eléctricos. Ambias empresas poseen altos niveles de desempeño y todas ellas son lideradas por su fundador Elon Musk. Su trabajo es traducir una visión empresarial en hechos concretos y medibles que favorece alcanzar logros de nivel mundial.

El liderazgo de los chilenos Daniel Undurraga, Juan Pablo Cuevas y el sueco Oskar Hjertonsson les permitió iniciar un emprendimiento con Cornershop, comprada por Uber, que recientemente se convierte en el primer Unicornio de Chile, esto es, una empresa valorada en torno a los 3.000 mil millones de dólares según Bloomberg (Munita, 2021).

La relevante participación de Mary T. Barra, ingeniera eléctrica y la primera mujer en asumir como presidenta ejecutiva de General Motors, ha sido todo un acontecimiento en una industria tradicionalmente liderada por hombres. Así, con su estilo tan particular de liderar ha sacado adelante a una empresa que participa de un mercado mundial altamente competitivo.

Ejemplos de liderazgo éxito al implementar la estrategia son numerosos, que al parecer todos ellos tiene una característica interesante, salen de la oficina (Bossidy \& Charan, 2002).

Uno de los últimos deseos de Steve Jobs fue la construcción del nuevo edificio corporativo de Apple, ubicado al sur de San francisco California. Este es circular, de 510 metros de diámetro y su costo fue de 5.000 millones de dólares. Lamentablemente, él no pudo ver concretada su obra. El especial diseño del edifico tanto en su exterior como en su interior, fue preocupación constante de Jobs, él sabía que la forma en que se organizan los recursos, sobre todo la forma en que interactúan los colaboradores de Apple tendría un impacto en la forma de ser y hacer de Apple. La lectura del libro de Isaacson (2011), muestra la vida de Steve Jobs y se pude concluir, entre muchas cosas, su especial cuidado y atención por el diseño de sus productos. En esa línea, también se logra visualizar su preocupación por la forma de organizarse para sacar adelante la estrategia de negocios de Apple. 
El diseño organizacional, es otro factor interactuante en la implementación de la estrategia (Chandler, 1990). En efecto, el diseño organizacional supuestamente sigue a la estrategia, aunque existen detractores a esta idea (Mintzberg, 2013). Sin embargo, determinar cuál debería ser el mejor diseño organizacional que acompañara la implementación de la estrategia es una tarea ardua no exenta de dificultades. En efecto, el diseño organizacional determinará cómo los recursos serán asignados, cuáles son las relaciones de autoridad, los niveles de jerarquía necesarios, entre tantas otras decisiones.

Steve Jobs, deseaba un diseño que permitiera las buenas comunicaciones, el trabajo en equipo y la fuerte interdependencia conversacional entre sus colaboradores que permita la construcción de una cultura de alto niveles de desempeño (Chandler, 1990). Los resultados, avalan su pensamiento. La cultura organizacional forma parte del conjunto de factores interdependientes que intervienen en la implementación de la estrategia, los aportes de autores relevantes como Kotter (1992) y Schein (2017) proveen un marco conceptual inicial para comprender como la cultura organizacional es vital para sacar adelante la estrategia.

Cuando se habla de cultura organizacional, se establece que los valores y creencias más profundas compartidos por los miembros de una organización están siempre presentes en el accionar diario de la empresa.

Por lo anterior, especial atención se debe tomar a este aspecto de la implementación estratégica. La cultura se gestiona y se mide (Denison \& Neale, 1996), y por tanto o la cultura está preparada para los cambios que exige la estrategia, o bien, la cultura deberá ser gestionada previamente para alcanzar los logros determinados por la estrategia.

La empresa Hewlett Packard, hoy HP Inc., se ha caracterizado por tener una cultura permeable a los grandes cambios que la industria electrónica demanda y ha logrado subsistir hasta hoy. Sin embargo, dicha cultura fue modelada pacientemente a lo largo del tiempo (Packard et al., 1995). En otras palabras, un cambio cultural organizacional no se logra de la noche a la mañana, de tal manera que muchas veces la estrategia formulada es excelente pero la cultura organizacional no está preparada para asumir el desafío de su implementación (Sull et al., 2015). Aquí, los éxitos pasados no necesariamente aseguran el éxito actual de la implementación de la nueva estrategia. Por ende, el aporte del área de personas y desarrollo organizacional se debe hacer presente en toda su magnitud.

El control estratégico que monitorea, desde una perspectiva proactiva más que reactiva, la marcha de la empresa es imprescindible para implementar la estrategia. La revisión de las desviaciones entre lo programado y el estado real de las cosas dan 
gobernabilidad al cambio estratégico.

Los sistemas ERP y los diferentes módulos de Business Intelligence (BI) permiten vincular el cuadro de mando integral de Norton y Kaplan (2008) en un ambiente que facilita la toma de decisiones en entornos complejos como los actuales. En ese contexto, la elección correcta de los indicadores claves de desempeño juega a favor de la, implementación de la estrategia de negocios (Paladino \& Williams, 2008).

Incentivos bien establecidos mueven y motivan el accionar de los colaboradores. En efecto, Los miembros individuales del equipo y los equipos tienen una necesidad fundamental de aceptación y apreciación y un sentido de agregar valor a cualquier organización a la que estén afiliados (Barth \& De Beer, 2018, p. 77).

Por lo anterior, la implementación de la estrategia debe venir acompañada con mecanismos que reconozcan el esfuerzo de los colaboradores y los equipos de trabajo en el cumplimiento de los hitos y metas necesarios para sacar adelante el trabajo comprometido.

Por supuesto, estos incentivos pueden ser monetarios y/o no monetarios. Pero, la idea de su existencia acompaña muy bien cualquier intención de implementar una estrategia de negocios. Finalmente, gestionar los factores que intervienen en la implementación de la estrategia no es la solución. Así, es la gestión interdependiente de dichos factores la que produce la sinergia suficiente como para sacar adelante lo descrito en el plan estratégico de la compañía. Todo un desafío.

\section{Conclusión}

Formular e implementar la estrategia de negocios son dos caras de una misma a moneda. En efecto, la organización busca obtener y mantener una ventaja competitiva. Para ello, necesita la intención y análisis que de sustento al diagnóstico estratégico que se traduce finalmente en una estrategia de negocio. Pero, de igual manera necesita procesos de toma de decisiones eficientes y eficaces que permitan llevar a la práctica lo escrito en un documento, el plan estratégico, a la realidad.

En dicho afán, existen factores que deben ser considerados para una implementación exitosa de la estrategia de negocios. Sin embargo, se debe tener en cuenta que son factores que aisladamente no se deben tratar. En efecto, reconocer la interdependencia de dichos factores permite "gestionar" la implementación de la estrategia de negocios con mejores resultados para la organización.

Durante el período 2020 y 2021, las organizaciones debieron adaptarse a las condiciones impuestas por medidas para contener una pandemia mundial. En ese 
contexto, el desafío de implementar exitosamente una estrategia de negocio fue particularmente difícil y de alto riesgo. Sin embargo, se lograron aprendizajes y buenas prácticas que afectaran positivamente las futuras implementaciones y adaptaciones de la estrategia del negocio.

Darwin planteaba que no es la más fuerte de las especies la que sobrevive, ni la más inteligente, sino la que mejor responde al cambio. Así, posiblemente la forma en que se deberá implementar la estrategia de negocios requerirá un cambio de enfoque.

\section{Referencias}

Barker, J. A. (1993). Paradigms : the business of discovering the future. HarperBusiness.

Barth, A. L., \& De Beer, W. (2018). Performance management success : a best practices and implementation guide for leaders and managers of all organizations. Springer.

Bennis, W. G., \& Nanus, B. (1997). Leaders : strategies for taking charge. HarperBusiness.

Bennis, W. G., \& Townsend, R. (2005). Reinventing leadership : strategies to empower the organization. Collins Business Essentials.

Bossidy, L., \& Charan, R. (2002). Execution : the discipline of getting things done. Crown Business.

Chandler, A. D. (1990). Strategy and structure : chapters in the history of the industrial enterprise. M.I.T. Press.

David, F. R., David, F. R., \& David, M. E. (2020). Strategic management : concepts and cases, a competitive advantage approach. Pearson.

Denison, D., \& Neale, W. S. (1996). Denison organizational culture survey : facilitator guide. Aviat.

Dess, G. G., McNamara, G., \& Eisner, A. B. (2019). Strategic management : creating competitive advantages. McGraw-Hill Education.

Drucker, P. F. (2017). The effective executive : the definitive guide to getting the right things done. HarperBusiness.

Favaro, K. (2015). Defining strategy, implementation, and execution. Harvard Business Review Digital Articles, 2-5. 
Gamble, J., Thompson, A. A., \& Peteraf, M. A. (2020). Essentials of strategic management : the quest for competitive advantage. McGraw-Hill Education.

Hill, C. W. L. (2019). Strategic management : theory \& cases. Cengage Learning.

Hitt, M. A., Ireland, R. D., \& Hoskisson, R. E. (2020). Strategic management : competitiveness \& globalization : concepts \& cases. Cengage.

Isaacson, W. (2011). Steve Jobs. Debate.

Kaplan, R. S., \& Norton, D. P. (1996). The balanced scorecard: translating strategy into action. Harvard Business School Press.

Kaplan, R. S., \& Norton, D. P. (2001). The strategy-focused organization : how balanced scorecard companies thrive in the new business environment. Harvard Business School Press.

Kaplan, R. S., \& Norton, D. P. (2004). Strategy maps : converting intangible assets into tangible outcomes. Harvard Business School Press.

Kaplan, R. S., \& Norton, D. P. (2006). Alignment : using the balanced scorecard to create corporate synergies. Harvard Business School Press.

Kaplan, R. S., \& Norton, D. P. (2008). The execution premium : linking strategy to operations for competitive advantage. Harvard Business Press.

Kaplan, R. S., Norton, D. P., Ferneau, P. J., High Performance Systems Inc., \& Harvard Business School Publishing Corporation. (1998). Balancing the corporate scorecard. In Harvard Business School Pub.

Kolbusa, M. (2013). Implementation management : high-speed strategy implementation. Springer.

Kotler, P., Berger, R., \& Bickhoff, N. (2008). The quintessence of strategic management : what you really need to know to survive in business. Springer.

Kotter, J. P. (2012). Leading change. Harvard Business Review Press.

Kotter, J. P., \& Heskett, J. L. (1992). Corporate culture and performance. Free Press.

Martin, R. L. (2010). The execution trap. Harvard Business Review, 88(7/8), 64-71.

Mintzberg, H. (2013). The strategy process : concepts, contexts, cases. Pearson Education. 
Mintzberg, H., Ahlstrand, B. W., \& Lampel, J. (2009). Strategy safari : the complete guide through the wilds of strategic management. FT Prentice Hall.

Munita, I. (2021, 22 Junio). Uber se hace del 100\% de Cornershop: la evolución que llevó a la startup chilena a convertirse en el primer "unicornio" del país. El Mercurio.

Packard, D., Kirby, D., \& Lewis, K. R. (1995). The HP way : how Bill Hewlett and I built our company. HarperBusiness.

Paladino, B., \& Williams, N. (2008). Moving strategy forward: merging the balanced scorecard and business intelligence. Business Performance Management, 6(2), 12-17.

Porter, M. E. (1980). Competitive strategy : techniques for analyzing industries and competitors. Free Press.

Porter, T. W., Harper, S. C., Glew, D. J., \& Aspley, J. C. (2007). Exceptional implementers. Industrial Management, 49(5), 14-19.

Radomska, J. (2014). The role of managers in effective strategy implememntation. International Journal of Contemporary Management, 13(3), 77-85.

Schein, E. H. (2017). Organizational culture and leadership. Wiley.

Sull, D., Homkes, R., \& Sull, C. (2015). Why strategy execution unravels- and what to do about It. Harvard Business Review, 93(3), 57-66.

Vyas, C., \& Westervelt, A. (2015). Why business transformation fails and how to ensure it doesn't. Forbes.com, 13-13.

Whetten, D. A., \& Cameron, K. S. (2020). Developing management skills. Pearson Education.

Ulises Bacho Gahona

Master of Business Administration, State University of New York, USA. Licenciado en Ciencias en la Administración de Empresas e Ingeniero Comercial, Universidad Católica del Norte, Chile. Certificado en Gobierno Corporativo, University of Michigan y Certificado en Planificación Estratégica Avanzada, University of California at Berkeley. Académico Núcleo Temático, Facultad de Administración, UNIACC, Chile.

Email: ulises.bacho@uniacc.edu 